\title{
The Boson peak in confined water: An experimental investigation of the liquid-liquid phase transition hypothesis
}

\author{
Francesco Mallamace ${ }^{1,2,3, *}$, Carmelo Corsaro ${ }^{2,3}$, Domenico Mallamace $^{4}$, Zhe Wang ${ }^{1}$, Sow-Hsin Chen ${ }^{1, \dagger}$ \\ ${ }^{1}$ Department of Nuclear Science and Engineering, Massachusetts Institute of Technology, Cambridge, MA 02139, USA \\ ${ }^{2}$ Dipartimento di Fisica e Scienze della Terra, Università di Messina, I-98166, Messina, Italy \\ ${ }^{3}$ Consiglio Nazionale delle Ricerche-IPCF Messina, I-98166, Messina, Italy \\ ${ }^{4}$ Dipartimento di Scienze dell'Ambiente, della Sicurezza, del Territorio, degli Alimentie, e della Salute, \\ Università di Messina Viale F. Stagno d'Alcontres 31, 98166 Messina, Italy \\ Corresponding authors.E-mail: *francesco.mallamace@unime.it, ${ }^{\dagger}$ sowhsin@mit.edu \\ Received July 6, 2015; accepted August 24, 2015
}

\begin{abstract}
The Boson peak (BP) of deeply cooled confined water is studied by using inelastic neutron scattering (INS) in a large interval of the $(P, T)$ phase plane. By taking into account the different behavior of such a collective vibrational mode in both strong and fragile glasses as well as in glass-forming materials, we were able to determine the Widom line that characterizes supercooled bulk water within the frame of the liquid-liquid phase transition (LLPT) hypothesis. The peak frequency and width of the BP correlated with the water polymorphism of the LLPT scenario, allowing us to distinguish the "low-density liquid" (LDL) and "high-density liquid" (HDL) phases in deeply cooled bulk water. Moreover, the BP properties afford a further confirmation of the Widom line temperature $T_{W}$ as the $(P, T)$ locus in which the local structure of water transforms from a predominately LDL form to a predominately HDL form.
\end{abstract}

Keywords supercooled water, liquid-liquid phase transition (LLPT), inelastic neutron scattering

PACS numbers 78.70.Nx, 64.70.Ja, 33.15.Fm

\section{Introduction}

Glassy materials play an important role in science and technology. Moreover, glasses and the corresponding supercooled liquids, from which glasses are usually prepared, have led to fundamental and challenging questions that have been the subject of a large number of studies in the past century. However, it is still not clear what happens when a liquid is cooled down to its amorphous glass phase [1]. Besides the anomalous increase of more than a factor of $10^{13}$ in the transport parameters (viscosity, self-diffusion, and relaxation times) that results in the continuous transition from a fluid to a glass, it is commonly accepted that two characteristic features dominate the behavior of glassy liquids as they evolve toward the glass state: i) the appearance of dynamic heterogeneities [2] and ii) the complicated energy landscape [3-7]. Just by observing the transport properties of a typical glass-forming supercooled liquid, it can be classified as being a strong (where the activation ener- gies are well defined) or a fragile liquid $[8,9]$; hence, a nearly Arrhenius temperature dependence is characteristic of a strong liquid, whereas a fragile liquid shows a quite non-Arrhenius behavior.

In the context of supercooled materials, water is of special interest and it presents intriguing and counterintuitive chemicophysical behaviors that have not been clearly explained [10]. However, it is well established from the various studies conducted on water that the hydrogen bond (HB) interactions between water molecules are the key to understand its properties and functions [11-13]. As $T$ decreases, the HB clusters form, leading to an open tetrahedrally coordinated HB network. This HB clustering explains the diverging behavior of various water thermal response and transport functions when the temperature is lowered to the supercooled region. More precisely, when the $T$ of the stable liquid phase is lowered, both the HB lifetime and cluster stability increase, and this altered local structure can, in principle, continue down to the amorphous region of the phase diagram. Amorphous water is polymorphic with low-density (LDA) and high-

*Special Topic: Water Science (Eds. Li-Mei Xu \& En-Ge Wang).

(C) The Author(s) 2015. This article is published with open access at www.springer.com/11467 
density (HDA) phases that can be transformed from one to the other by varying the pressure [14].

This polymorphism of glassy water and the HB networking suggests that liquid water can also be polymorphous, i.e., a mixture of a low-density liquid (LDL) and a high-density liquid (HDL). In the HDL form, which predominates in the high- $T$ regime, the local tetrahedrally coordinated structure is not fully developed, but in the LDL form, a more open "ice-like" HB network appears. Thus, water's anomalies are caused by the "competition" between these two local liquid forms. At ambient pressure, metastable supercooled water is located on the phase diagram between the melting temperature $T_{M}=273 \mathrm{~K}$ and the homogeneous nucleation temperature $T_{H}=231 \mathrm{~K}$; below $T_{g} \approx 130 \mathrm{~K}$, water exists as glass; above this temperature, it becomes a highly viscous fluid that crystallizes at $T_{X} \approx 150 \mathrm{~K}$. The region between $T_{H}$ and $T_{X}$ is not experimentally accessible to bulk liquid water and is referred to as the "No-Man's Land" of the phase diagram. The crystallization within this latter region (i.e., the no-man's land) can be retarded to some extent by confining water within the narrow nanoporous structures. Studies on confined water $[15,16]$ have shown that the water HB networking and the HB lifetime greatly increase on decreasing the temperature, indicating the presence of a dynamic crossover from a fragile-to-strong liquid (FSDC) at $T_{L} \simeq 225 \mathrm{~K}$ and ambient pressure. At this temperature, clear signs of LDL and HDL are observed [16], confirming that polymorphism dominates the water liquid phase; it also supports the liquid-liquid phase transition (LLPT) hypothesis [18]. In addition, the violation of the Stokes-Einstein relation is also observed [16, 17]. Furthermore, within the framework of the LLPT model, the so-called Widom line (the LDL-HDL coexistence line) is important. It identifies the locus, $T_{W}$, of the maximum correlation length, where the thermodynamic response function have an extrema (maximum or minimum) [19, 20]. On decreasing $T$, the Widom line must converge to the liquid-liquid critical point C', where the correlation length diverges; hence, the end point of the Widom line is the water's hypothesized second critical point. These experimental observations on the water FSDC, including the findings of neutron experiments in a wide $P-T$ interval of the water phase diagram [18], confirm that the FSDC locus just identifies the Widom line so that $T_{L} \equiv T_{W}$. The water $P-T$ phase diagram proposed for bulk water according to the polymorphism in the LLPT frame is illustrated in Fig. 1.

The FSDC, with $T_{L}>T_{g}$, is not limited to water but also characterizes other supercooled glass-forming liquids $[6,7,9,21,23]$. Its appearance indicates that the sys-

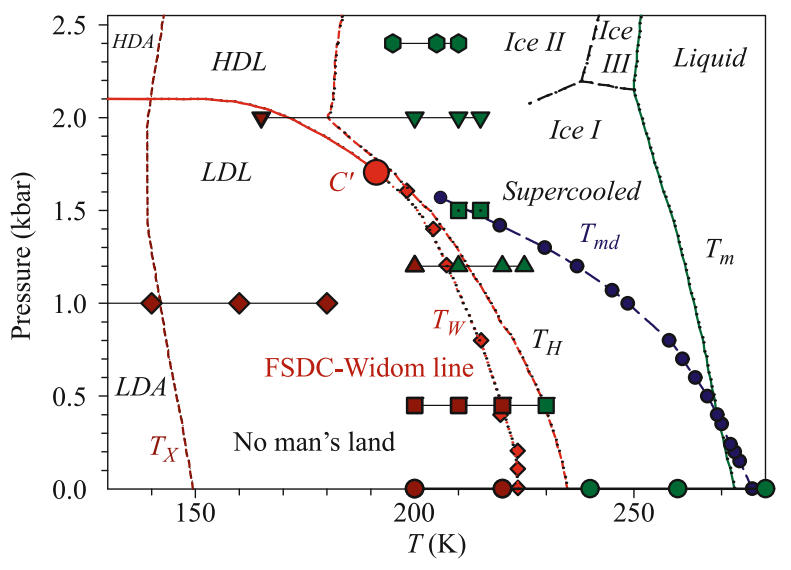

Fig. 1 The pressure-temperature phase diagram of bulk water in the frame of its polimorphism and of the LLPT model. The LDA, the HDA, the LDL and HDL phases and the Widom line (the locus of $T_{W}$, dotted line) are reported together with the melting $T_{m}$, the homogeneous nucleation temperature $T_{H}$ and the crystallization $T_{X}$ loci. The LL critical point C' and the line of the density maxima, $T_{m d}$, are also reported. Dark red and green large symbols, as illustrated in the text, deal with the findings of this work.

tem is approaching the dynamic arrest $[6,7,9,21,22$, 37]; many special processes occur at the dynamic arrest (at FSDC) (see, e.g., Ref. [9]), including the violation of the Stokes-Einstein law [2], orientational-translational decoupling [4], splitting of the relaxation into primary $(\alpha)$ and secondary relaxation times $(\beta)$, onset of dynamic heterogeneities, and onset of the Boson peak (BP) $[2,24-$ $26]$.

The BP, one of the most intriguing properties of supercooled liquids and glasses, is usually observed in the terahertz region by Raman or inelastic neutron scattering experiments [27-33]. The BP, a collective mode, appears as an excess over the density of vibrational states, $g(E) \propto E^{2}$ predicted by the Debye model and is probably the most intensively studied feature of amorphous materials. Despite this and the BP ubiquities in disordered systems, the identification of the physical origin of $\mathrm{BP}$ is unclear. Several different models, from harmonic excitations of a disordered lattice [34] to mosaic structures related to dynamic heterogeneities and the energy landscape [26], have been proposed to explain the physical mechanism of BP. There is a growing consensus on some BP properties: i) the BP frequency (or energy $E$ ) corresponds to the maximum frequency at which transverse phonons can propagate in the disordered material [36] (i.e., the Ioffe-Regel limit); ii) the BP intensity increases with a decrease in the fragility, hence systems with a strong BP are those with "strong" liquid character, whereas fragile glass formers have weak BPs [35, $36]$.

This latter situation that regards the BP characteristic of low-frequency excitations and the slowest transport 
behavior (another intriguing property of glass-forming materials) is studied here. In fact, we study the BP at the water FSDC in order to verify if there is any link between the BP and the Widom line as recently hypothesized for bulk water by an MD simulation study [37] and observed in confined water $[38,39]$. In other words, the aim of the present work, by using inelastic neutron scattering (INS), is to verify in the $(P, T)$ plane whether deeply cooled water confined in nanopores can provide confirmation about the appearance of LLPT.

\section{Experiments}

We report a BP data analysis of INS spectra reported in our two previous papers [38, 39]. The INS experiments were carried out in the following $P-T$ ranges: $120 \mathrm{~K}<T<230 \mathrm{~K}$ and 1 bar $<P<2400$ bar (large symbols in Figure 1). More precisely, for $P=1$ bar we used the HRMECS chopper at the Intense Pulsed Neutron Source (IPNS) at the Argonne National Laboratory, whereas for the other data we used the Disk Chopper Spectrometer (DCS) at the National Institute of Standards and Technology (NIST) Center for Neutron Research and the Cold Neutron Chopper Spectrometer (CNCS) at the Spallation Neutron Source of the Oak Ridge National Laboratory (ORNL). In order to

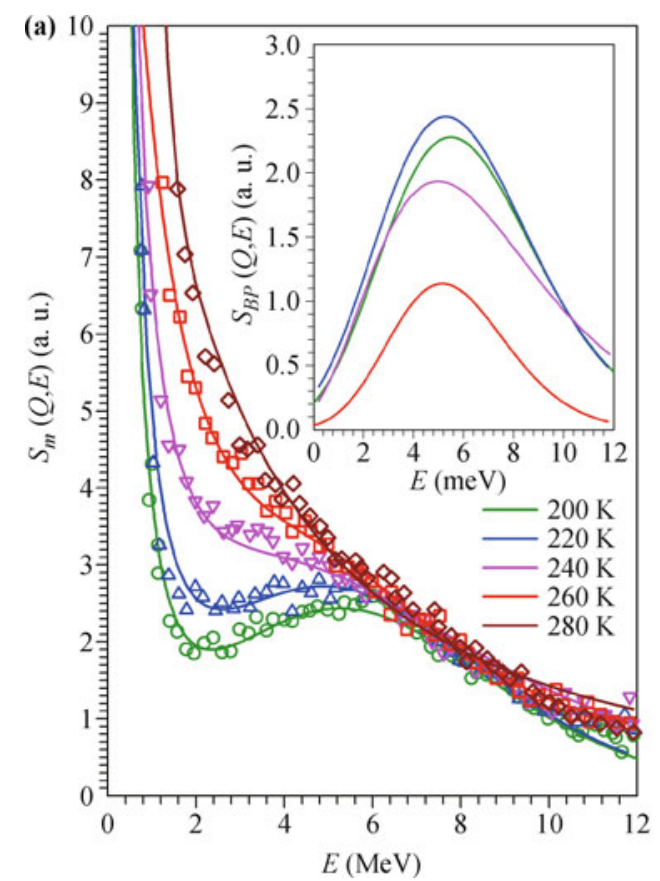

enter the deeply supercooled region of water, we used a nanoporous silica matrix, MCM-41-S, with $15 \AA$ pore diameter to confine water. Under this confinement, water remains in the liquid state at temperatures much lower than that of homogeneous nucleation. Details on the experimental procedure and sample preparation are reported elsewhere $[38,39]$. The measured energy spectra for a fixed wavevector $Q$ (the magnitude of the momentum transfer of the incident neutron) are indicated as $S_{m}(Q, E)$. Figure 2(a) reports the self-dynamic structure factor, $S_{m}(Q, E)$, of the confined water measured for $P=1$ bar at $T=200,220,240,260$, and $280 \mathrm{~K}$. A $\mathrm{BP}$ is clearly observable for $E \simeq 5.5 \mathrm{meV}$ at $T=200$ and $220 \mathrm{~K}$, hence near the water FSDC or Widom temperature, whereas above $220 \mathrm{~K}$, the BP gradually merges into the quasielastic contribution.

All the spectral contributions $S_{m}(Q, E)$, measured at a fixed $Q$, were analyzed by taking into account the energy resolution function $(R(E))$ by means of a Lorentzian (the quasi-elastic contribution) function and a log-normal distribution (LND) as

$$
\begin{gathered}
S_{m}(Q, E)=R(E) \otimes\left\{\left[\frac{A_{1} \gamma_{1}}{\pi\left(E^{2}+\gamma_{1}^{2}\right)}\right.\right. \\
\left.\left.+\frac{A_{2}}{\sqrt{\pi} \sigma_{B P} E} \exp \left(-\frac{\left(E_{B P}-\ln E\right)^{2}}{2 \sigma_{B P}^{2}}\right)\right] D(E)\right\}
\end{gathered}
$$

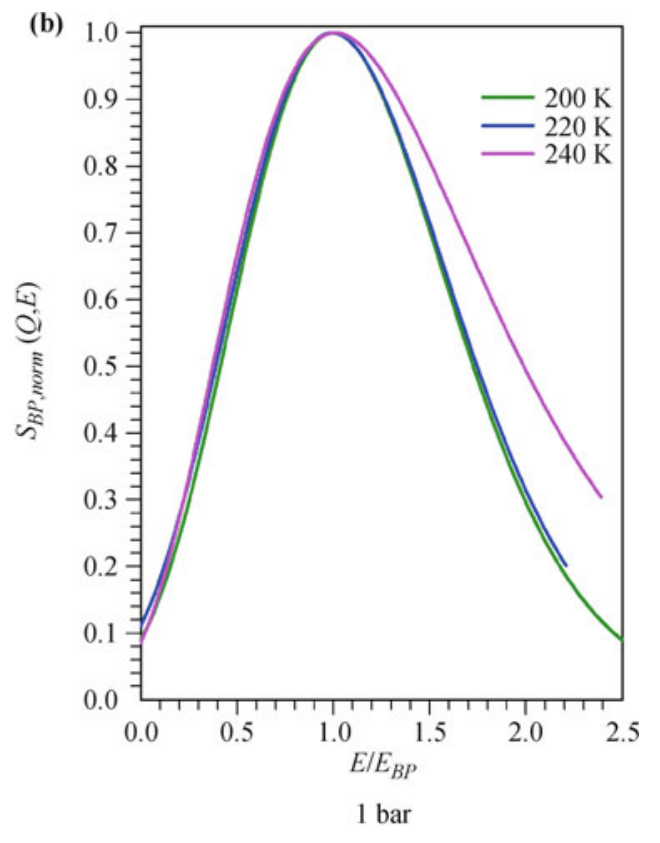

Fig. 2 (a) The Inelastic Neutron Scattering (INS) spectra at $P=1$ bar of the MCM-41 confined water $S_{m}(Q, E)$ measured at $200,220,240,260$, and $280 \mathrm{~K}$. The presence of the Boson peak (BP) is evident for $T \leqslant 260 \mathrm{~K}$. The spectra evaluated according to the Eq. (1) are reported as continuous line $\left(S_{t h}(Q, E)\right)$. In the inset the obtained BP spectra $S_{B P}(Q, E)$ are reported. (b) shows the normalized BP spectral contributions, $S_{B P, \text { norm }}(Q, E)$, for $T=200,220$, and $240 \mathrm{~K}$. As it can be observed the contribution at $240 \mathrm{~K}$ (inside the supercooled "fragile" HDL phase $T>T_{H}>T_{W}$ ) is broader than those corresponding to the "strong" LDL dominant phase located below the Widom line $\left(T=200,220 \mathrm{~K}\right.$. i.e., $\left.T<T_{W}\right)$. 
where $\sigma_{B P}$ and $E_{B P}$ relate to the $\mathrm{BP}$ width and position, respectively; $A_{1}$ and $A_{2}$ are the amplitudes of these two parts; and $D(E)$ is the detailed balance factor and is expressed as $\exp \left(E /\left(2 k_{\mathrm{B}} T\right)\right)$. The LND well describes the $\mathrm{BP}$ and has been successfully applied to confined water [40].

\section{Results and discussion}

The left side of Fig. 2(a) illustrates the BP contribution of confined water, $S_{B P}(Q, E)$, evaluated from $S_{m}(Q, E)$ at $P=1$ bar, where $T_{W} \simeq 225 \mathrm{~K}$. The continuous lines in Fig. 2(a) represent the fit, $S_{t h}(Q, E)$, of the corresponding data, giving a good representation of the quality of the data analysis in terms of Eq. (1). The corresponding inset reports the evaluated $\mathrm{BP}$ contribution $\left(S_{B P}(Q, E)\right)$. As it can be observed, the BP strength shows a marked increase on decreasing $T$ up to the region of $T_{W}$. Below $T_{W}$, it evolves $(T=220$ and $200 \mathrm{~K})$ with a different rate; at the same time, a spectral narrowing and a shift in the frequency value of the BP maximum $\left(E_{B P}\right)$ can be observed. The BP physical evolution is clearer if we consider a normalized representation of $S_{B P}(Q, E)$ versus $E_{B P}$ and the peak strength. In this case from the normalized BP spectra, $S_{B P, \text { norm }}(Q, E)$ [Fig. 2(b)], a narrowing in the BP evolution is well evident when the supercooled liquid water crossovers from a fragile to a strong "status" of the glass former. This is an expected situation considering the results of various studies on BP dependence as a function of fragility, but it assumes a more important meaning if we consider that the Widom line also represents a structural crossover from a predominantly HDL structure to a predominantly LDL structure [15, 41]. From the dynamic point of view, the water $T_{W}$ also represents the locus in which the Stokes-Einstein law is violated [16] according to the orientational-translational decoupling [4] due to HB networking and change in the energetic configurations [3-7]. More precisely, the FSDC phenomenon in the energy landscape framework (inherent structures) is interpreted as system evolution, by decreasing $T$, from multibasin dynamics to molecular trapping in deep energy basins.

Starting from these results at $P=1$ bar, we have considered a more complete study by exploring other pressures in order to verify if or not the obtained findings were confirmed. Figures 3(a) and 4(a) report the $S_{t h}(Q, E)$ obtained by means of the same data analysis for the INS spectra measured at 450 bar $(T=200,210$, 220 , and $230 \mathrm{~K})$ and $1.2 \mathrm{kbar}(T=200,210,220$, and 225 $\mathrm{K})$, respectively. The BP behavior for these two pressures is similar to that observed in the 1 bar INS spectra (see the insets of the corresponding figures). From Figs. 3(b) and 4(b), where the normalized BP spectra are reported, the main result obtained for the 1 bar INS spectra is fully confirmed; i.e., the BP spectrum changes on crossing the Widom line, showing a narrowing just when entering the LDL phase. We have also measured the BP at the following additional pressures: 1, 1.5,2, and $2.4 \mathrm{kbar}$ by considering, respectively, the following temperatures: 140, 160, and $180 \mathrm{~K}$ (1 kbar); 215 and $220 \mathrm{~K}$ (1.5 kbar); $165,200,210$, and $215 \mathrm{~K}$ (2 kbar); and finally 195, 205, and 210 at $2.4 \mathrm{kbar}$. In the first case (1 kbar), all the three temperatures of the measured INS spectra lie in the LDL region, whereas the values of 1.5 and $2.4 \mathrm{kbar}$ are localized outside the no man's land and in particular in the supercooled liquid region dominated by the HDL phase. The same holds for the highest temperatures at 2 kbar, except for $T=165 \mathrm{~K}$, a temperature less than that of the second critical point $\mathrm{C}^{\prime}$, and at the border line between the LDL and HDL phases. All these explored temperatures in which the BP is clearly observed from the INS spectra, $S_{m}(Q, E)$, are shown as large symbols in Fig. 1. More precisely, the dark red symbols identify the narrow BP inside the LDL phase (below the Widom line, i.e., $T<T_{W}$ ), whereas the green symbols are all localized in the supercooled liquid region. Such a situation is well accounted in Fig. 5, where the values of the half width at half maximum $\left(\mathrm{HWHM}_{B P, \text { norm }}\right)$ of the BP normalized spectra $S_{B P, \text { norm }}(Q, E)$ studied herein are reported as a function of temperature. In particular, all the data inside the LDL region, i.e., corresponding to temperatures and pressures for $T<T_{W}$, are reported as dark red symbols, whereas the others are reported as green symbols. As it can be observed, the data of BP spectra measured in the LDL region cover a completely different area of Fig. 5 when compared with those measured inside the supercooled region. More precisely, a sort of crossover can be located at about 1.35, between the reported $\mathrm{HWHM}_{B P, \text { norm }}$ values. In particular, below it fall all the BP spectra belonging to the more structured configurations, in terms of the HB tetrahedrally coordinated network, i.e., the region of dominance of the LDL phase, whereas the BP spectral contributions of the LDL region fall above this value. From Fig. 5, it can be noticed that the spread in the $\operatorname{HWHM}_{B P \text {, norm }}$ values inside the LDL -dominated phase has a very limited range, 1.2-1.35, whereas the corresponding quantity of the data within the HDL region, above the Widom line, is much larger, ranging from 1.35 to more than 1.7. If we go back to the starting considerations that the LDLdominated region is the "strong" glass-forming region, contrary to the HDL that is "fragile", then the obtained 

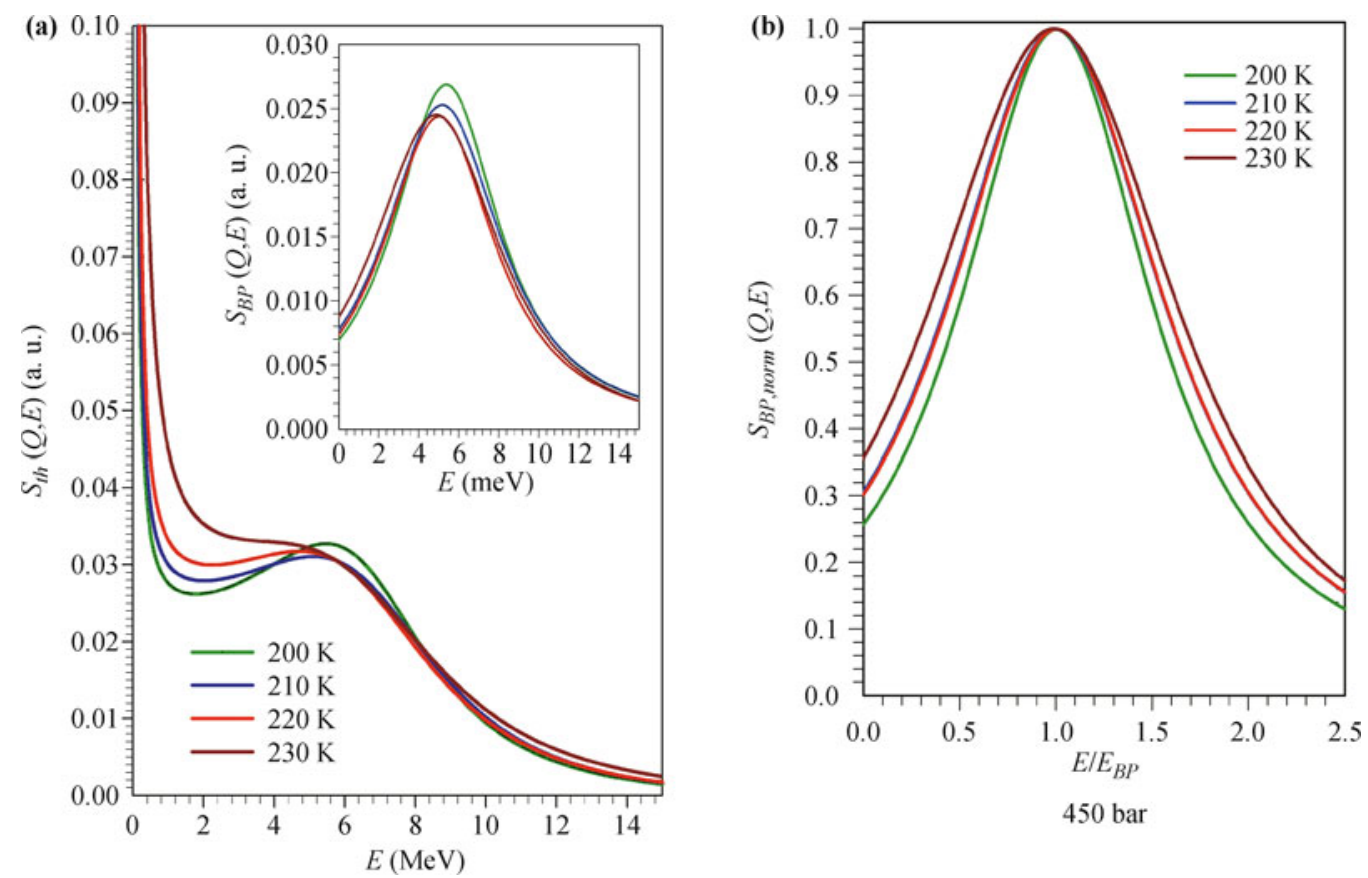

Fig. 3 (a) The fitted Inelastic Neutron Scattering (INS) spectra for the MCM-41 confined water $S_{t h}(Q, E)$ at $P=450$ bar measured at 200,210, 220, and $230 \mathrm{~K}$. The inset illustrates the corresponding BP spectra $S_{B P}(Q, E)$. (b) reports the corresponding $S_{B P, n o r m}(Q, E)$. In this case according to the used procedure, the Widom line is localized at about $T_{W}=220$ $\mathrm{K}$; in fact, the obtained $S_{B P, \text { norm }}$ values are broader for the higher temperature and narrower for lower temperatures.
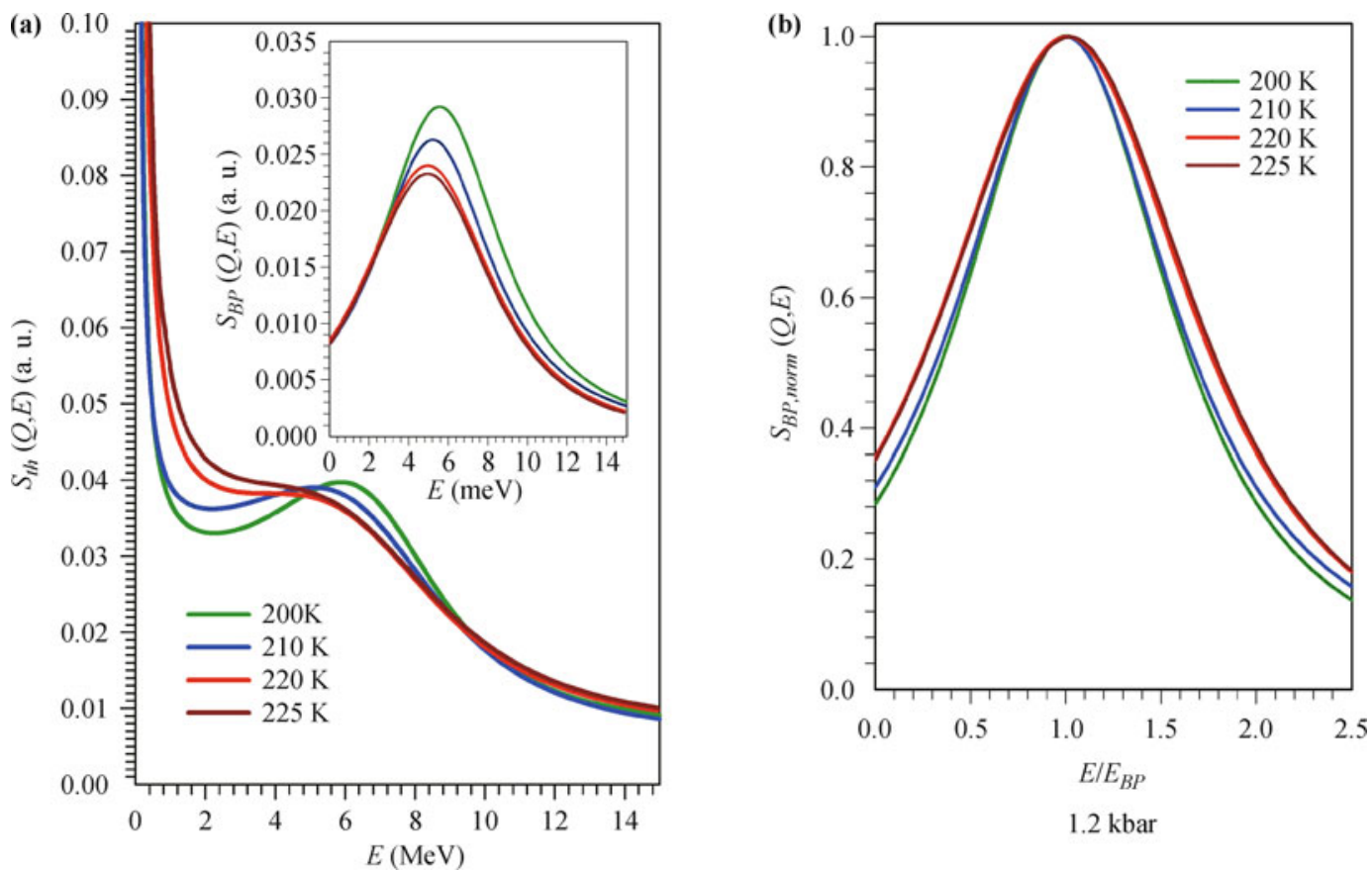

$1.2 \mathrm{kbar}$

Fig. 4 The fitted Inelastic Neutron Scattering (INS) spectra for the MCM-41 confined water $S_{t h}(Q, E)$ at $P=1.2 \mathrm{kbar}$ measured for $T=200,210,220$, and $225 \mathrm{~K}$. Also in this case the obtained findings are in good agreement with the previous results corresponding to $P=1$ bar and 450 bar. In the present case the Widom line seems to be located between 200 and $210 \mathrm{~K}$.

findings fully agree with the observed BP behavior in other glasses and glass-forming liquids $[2,24-26,35,36]$; i.e., the corresponding spectra are more localized for the strong materials, whereas they are broadly distributed for the fragile materials.

\section{Conclusion}

We have considered the experimental INS spectra of confined water in order to characterize its structural and 


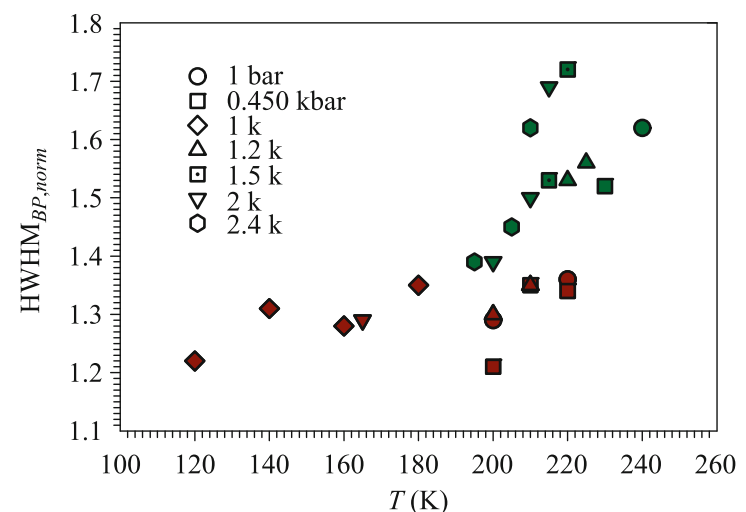

Fig. 5 The HWHM values of the normalized BP $\left(\mathrm{HWHM}_{B P, \text { norm }}\right)$ measured for all the studied pressures (range 1 bar $<P<2.4$ kbar) reported as a function of the temperature in the range $100 \mathrm{~K}<T<260 \mathrm{~K}$. As it can be observed, the data measured above (green symbols) and below (dark red symbols) the Widom line are localized in separate different areas of the plot.

dynamic evolution by studying the properties of the socalled BP, the well-known collective low-frequency vibrational mode characterizing amorphous glasses and supercooled liquids. We took advantage of a universal-like behavior observed for the BP width; the peak frequency results showed marked differences between those of strong and fragile glasses and glass-forming systems during a thermodynamic change in their $P-T$ phase $[2,24-26,35$, $36]$. In simple words, based on the explanation of the energy landscape model, the BP width of strong glasses is narrower $[26,35,36]$ and less $T-P$ dependent when compared with the corresponding $\mathrm{BP}$ width of fragile glasses [42].

A recent MD study [37] has proposed "that the onset of the Boson peak in supercooled bulk water coincides with the crossover to a predominantly low-density-like liquid below the Widom line $T_{W}$." In addition, it has been stressed that the frequency and onset temperature of the BP of bulk water agree well with the results obtained from experiments on nanoconfined water [38]. Such a picture has been essentially confirmed by an accurate INS study [39]. Here, by considering the abovementioned "universal-like behavior" of the BP, we essentially confirm the link between the BP and the water polymorphism, i.e., the BP connection with the "LDL" and "HDL" phases in deeply cooled water. Moreover, we highlight that the BP onset is localized not at the Widom line, but inside the fragile region of water, just above the homogeneous nucleation temperature $T_{H}=231 \mathrm{~K}$ in a $P-T$ region dominated by a large concentration of the HDL with respect to the LDL phase. We conclude by stressing that the Widom line is detectable by changes in the BP frequency and width of the crossover from the fragile to the strong glass-forming region; i.e., on ap- proaching a phase region dominated by the LDL phase.

Acknowledgements The research at MIT of SHC and ZW was supported by DOE grant DE-FG02-90ER45429.

Open Access This article is distributed under the terms of the Creative Commons Attribution License which permits any use, distribution, and reproduction in any medium, provided the original author(s) and the source are credited.

\section{References}

1. D. Kennedy, 125th Anniversary Issue: 125 outstanding problems in all of science: What is the nature of the glassy state, Science 309, 83 (2005)

2. M. D. Ediger, Spatially heterogeneous dynamics in supercooled liquids, Annu. Rev. Phys. Chem. 51(1), 99 (2000)

3. M. Goldstein, Viscous liquids and the glass transition: A potential energy barrier picture, J. Chem. Phys. 51(9), 3728 (1969)

4. F. H. Stillinger, A topographic view of supercooled liquids and glass formation, Science 267(5206), 1935 (1995)

5. A. Laio and M. Parrinello, Escaping free-energy minima, Proc. Natl. Acad. Sci. USA 99(20), 12562 (2002)

6. F. Mallamace, C. Branca, C. Corsaro, N. Leone, J. Spooren, S. H. Chen, and H. E. Stanley, Transport properties of glassforming liquids suggest that dynamic crossover temperature is as important as the glass transition temperature, Proc. Natl. Acad. Sci. USA 107(52), 22457 (2010)

7. S. Yip and M. P. Short, Multiscale materials modeling at the mesoscale, Nat. Mater. 12(9), 774 (2013)

8. C. A. Angell, Formation of glasses from liquids and biopolymers, Science 267(5206), 1924(1995)

9. J. C. Martinez-Garcia, J. Martinez-Garcia, S. J. Rzoska, and J. Hulliger, The new insight into dynamic crossover in glass forming liquids from the apparent enthalpy analysis, $J$. Chem. Phys. 137(6), 064501 (2012)

10. Advances in Chemical Physics, Water Polymorphism, edited by H. E. Stanley, series editor S. A. Rice, Wiley, New York, 2013

11. F. Mallamace, P. Baglioni, C. Corsaro, J. Spooren, H. E. Stanley, and S.H. Chen, Transport properties of supercooled confined water, Riv. Nuovo Cim. 34, 253 (2011)

12. F. Mallamace, C. Corsaro, D. Mallamace, C. Vasi, and H. E. Stanley, The thermodynam-ical response functions and the origin of the anomalous behavior of liquid water, Farad. Disc. 167, 95 (2013)

13. F. Mallamace, C. Corsaro, and H. E. Stanley, A singular thermodynamically consistent temperature at the origin of the anomalous behavior of liquid water, Sci. Rep. 2, 993 (2012)

14. O. Mishima, Relationship between melting and amorphization of ice, Nature 384(6609), 546 (1996) 
15. F. Mallamace, M. Broccio, C. Corsaro, A. Faraone, D. Majolino, V. Venuti, L. Liu, C. Y. Mou, and S. H. Chen, Evidence of the existence of the low-density liquid phase in supercooled, confined water, Proc. Natl. Acad. Sci. USA 104(2), 424 (2007)

16. S. H. Chen, F. Mallamace, C. Y. Mou, M. Broccio, C. Corsaro, A. Faraone, and L. Liu, The violation of the StokesEinstein relation in supercooled water, Proc. Natl. Acad. Sci. USA 103(35), 12974 (2006)

17. L. Xu, F. Mallamace, Z. Yan, F. W. Starr, S. V. Buldyrev, and H. E. Stanley, Appearance of a fractional Stokes Einstein relation in water and a structural interpretation of its onset, Nat. Phys. 5(8), 565 (2009)

18. P. H. Poole, F. Sciortino, U. Essmann, and U. H. E. Stanley, Phase behaviour of metastable water, Nature 360(6402), 324 (1992)

19. L. Liu, S. H. Chen, A. Faraone, C. Yen, and C. Y. Mou, Pressure dependence of fragile-to-strong transition and a possible second critical point in supercooled confined water, Phys. Rev. Lett. 95(11), 117802 (2005)

20. L. Xu, P. Kumar, S. V. Buldyrev, S.H. Chen, P. H. Poole, F. Sciortino, and H. E. Stanley, Relation between the Widom line and the dynamic crossover in systems with a liquidliquid phase transition, Proc. Natl. Acad. Sci. USA 102(46), 16558 (2005)

21. J. C. Mauro, Y. Yue, A. J. Ellison, P. K. Gupta, and D. C. Allan, Viscosity of glass-forming liquids, Proc. Natl. Acad. Sci. USA 106(47), 19780 (2009)

22. F. Mallamace, C. Corsaro, H. E. Stanley, and S. H. Chen, The role of the dynamic crossover temperature and the arrest in glass-forming fluids, Eur. Phys. J. E 34(9), 94 (2011)

23. T. Hecksher, A. I. Nielsen, N. B. Olsen, and J. C. Dyre, Little evidence for dynamic divergences in ultraviscous molecular liquids, Nat. Phys. 4(9), 737 (2008)

24. M. D. Ediger, C. A. Angell, and S. R. Nagel, Supercooled liquids and glasses, J. Phys. Chem. 100(31), 13200 (1996)

25. M. D. Ediger and P. Harrowell, Perspective: Supercooled liquids and glasses, J. Chem. Phys. 137(8), 080901 (2012)

26. V. Lubchenko and P. Wolynes, Theory of structural glasses and supercooled liquids, Annu. Rev. Phys. Chem. 58(1), 235 (2007)

27. B. Frick and D. Richter, The microscopic basis of the glass transition in polymers from neutron scattering studies, Science 267(5206), 1939 (1995)

28. V. N. Novikov and A. P. Sokolov, A correlation between lowenergy vibrational spectra and first sharp diffraction peak in chalcogenide glasses, Solid State Commun. 77(3), 243 (1991)

29. V. K. Malinovsky, V. N. Novikov, P. P. Parshin, A. P. Sokolov, and M. G. Zemlyanov, Universal form of the lowenergy (2 to $10 \mathrm{meV}$ ) vibrational spectrum of glasses, Europhys. Lett. 11(1), 43 (1990)
30. A. P. Sokolov, U. Buchenau, W. Steffen, B. Frick, and A. Wischnewski, Comparison of Raman- and neutronscattering data for glass-forming systems, Phys. Rev. B 52(14), R9815 (1995)

31. J. Wuttke, J. Hernandez, G. Li, G. Coddens, H. Z. Cummins, F. Fujara, W. Petry, and H. Sillescu, Neutron and light scattering study of supercooled glycerol, Phys. Rev. Lett. 72(19), 3052 (1994)

32. B. Hehlen, E. Courtens, R. Vacher, A. Yamanaka, M. Kataoka, and K. Inoue, Hyper-Raman scattering observation of the Boson peak in vitreous silica, Phys. Rev. Lett. 84(23), 5355 (2000)

33. J. Wuttke, M. Kiebel, E. Bartsch, F. Fujara, W. Petry, and H. Sillescu, Relaxation and phonons in viscous and glassy orthoterphenyl by neutron scattering, Z. Phys. B 91(3), 357 (1993)

34. S. Grigera, V. Mart'ın-Mayor, G. Parisi, and P. Verrocchio, Vibrational spectrum of topologically disordered systems, Phys. Rev. Lett. 87(8), 085502 (2001)

35. A. P. Sokolov, R. Calemczuk, B. Salce, A. Kisliuk, D. Quitmann, and E. Duval, Low-temperature anomalies in strong and fragile glass formers, Phys. Rev. Lett. 78(12), 2405 (1997)

36. H. Shintani and H. Tanaka, Universal link between the boson peak and transverse phonons in glass, Nat. Mater. 7(11), 870 (2008)

37. P. Kumar, K. T. Wikfeldt, D. Schlesinger, L. G. M. Pettersson, and H. E. Stanley, The Boson peak in supercooled water, Sci. Rep. 3, 1980 (2013)

38. S. H. Chen, Y. Zhang, M. Lagi, S. H. Chong, P. Baglioni, and F. Mallamace, Evidence of dynamic crossover phenomena in water and other glass-forming liquids: experiments, MD simulations and theory, J. Phys.: Condens. Matter 21(50), $504102(2009)$

39. Z. Wang, K. H. Liu, P. Le, M. Li, W. S. Chiang, J. B. Leão, J. R. D. Copley, M. Tyagi, A. Podlesnyak, A. I. Kolesnikov, C.Y. Mou, and S. H. Chen, Boson peak in deeply cooled confined water: A possible way to explore the existence of the liquid-to-liquid transition in water, Phys. Rev. Lett. 112(23), 237802 (2014)

40. A. Cupane, M. Fomina, and G. Schirò, The boson peak of deeply cooled confined water reveals the existence of a low-temperature liquid-liquid crossover, J. Chem. Phys. 141, 18C510 (2014).

41. K. T. Wikfeldt, A. Nilsson, and L. G. M. Pettersson, Spatially inhomogeneous bimodal inherent structure in simulated liquid water, Phys. Chem. Chem. Phys. 13(44), 19918 (2011)

42. L. Hong, B. Begen, A. Kisliuk, C. Alba-Simionesco, V. N. Novikov, and A. P. Sokolov, Pressure and density dependence of the Boson peak in polymers, Phys. Rev. B 78(13), 134201 (2008) 